\title{
Pré-operatório na sala de recuperação pós-anestésica: 0 ambiente pode influenciar?
}

The pre-operative period in the post-anesthetic recovery room: does the environment influence the patient?

Preoperatorio en la sala de recuperación post-anestésica: ¿el ambiente puede influenciar?

Letícia Silva de Araújo ${ }^{1}$, Poliana Rodrigues Vilas Boas ${ }^{2}$, Leila de Fátima Santos ${ }^{3}$, Lilian Machado Torres ${ }^{4}$

\section{Resumo}

A Sala de Recuperação Pós-Anestésica é um local destinado à recuperação de pacientes que já passaram por procedimentos cirúrgicos. No entanto, é comum que os indivíduos em período pré-operatório permaneçam nesse ambiente, gerando um convívio desnecessário que pode aumentar a ansiedade e o estresse dos que ainda serão submetidos ao procedimento cirúrgico. Os objetivos do estudo foram compreender a percepção do paciente que aguarda a sua cirurgia no mesmo espaço destinado aqueles submetidos a procedimentos cirúrgicos, e propor melhorias que visem à manutenção do

1 Enfermeira. Especializanda em Neonatalogia. Minas Gerais, Brasil. E-mail: lele_silvaaraujo@hotmail.com

2 Enfermeira.Especializanda em Trauma, Emergência e Terapia Intensiva. Enfermeira auditora na Maternidade Hospital Octaviano Neves. Belo Horizonte- MG, Brasil. Email: polianarv@hotmail.com

${ }^{3}$ Enfermeira. Mestre em Administração. Docente no Curso de Enfermagem da Faculdade Ciências Médicas de Minas Gerais. Minas Gerais, Brasil. E-mail: leila.santos@cienciasmedicasmg.edu.br

${ }^{4}$ Doutora em Ciências da Saúde pela USP (2015). Docente no Curso de Enfermagem da Faculdade Ciências Médicas de Minas Gerais e Enfermeira do Instituto de Previdência dos Servidores do Estado de Minas Gerais. Minas Gerais, Brasil. E-mail: lilian.torres@ superig.com.br bem-estar e a garantia da qualidade da assistência prestada no ambiente cirúrgico. Pesquisa de natureza exploratória, com abordagem qualitativa que entrevistou 15 pacientes. Os resultados evidenciaram sentimentos relacionados à indiferença; incômodo; tranquilidade; felicidade por certificarem-se do sucesso; e comoção pela dor do outro. Torna-se importante que as equipes cirúrgicas conheçam tais sentimentos e reflitam permanentemente, no sentido da qualificação do cuidado perioperatório. Mudanças estruturais e de processo também podem contribuir para o acolhimento mais cuidadoso do paciente em ambiente cirúrgico.

Descritores: Enfermagem. Enfermagem de Centro Cirúrgico. Sala de Recuperação. Cuidados pré-operatórios.

\section{Abstract}

The post-anesthetic recovery room is a place for the recovery of patients who have undergone surgical procedures. However, it is common that preoperative period individuals remain 
in that environment, generating an unnecessary experience that can increase anxiety and stress of those who will still undergo their surgical procedure. The objectives of this study were to understand the perception of patients who are awaiting surgery in the same space as those who have already undergone surgical procedures, and propose improvements aimed at maintaining well-being and ensuring quality of assistance provided in the operating environment. This was a qualitative exploratory research that interviewed 15 patients. The results showed feelings related to indifference; feeling bothered/uncomfortable; calmness; happiness from seeing (surgical) success; and turmoil from the suffering of others. It is important that surgical teams are aware of such feelings and constantly reflect on the qualification of perioperative care. Structural and procedural changes could also contribute to more careful care of the patient in the surgical environment.

Descriptors: Nursing. Operating Room Nursing. Recovery Room. Preoperative care.

\section{Resumen}

La Sala de Recuperación PostAnestésica es un lugar destinado a la recuperación de pacientes que ya pasaron por procedimientos quirúrgicos. Sin embargo, es común que las personas en período preoperatorio permanezcan en ese ambiente, generando una convivencia innecesaria que puede aumentar la ansiedad y el estrés de quienes todavía deben ser sometidos al procedimiento quirúrgico. El objetivo del estudio es comprender la percepción del paciente que aguarda su cirugía en el mismo lugar destinado a aquellos que ya fueron sometidos a procedimientos quirúrgicos y proponer mejoras orientadas a mantener el bienestar y a garantizar la calidad de la asistencia brindada en el ambiente quirúrgico. Investigación de naturaleza exploratoria con un enfoque cualitativo que entrevistó a 15 pacientes. Los resultados evidenciaron sentimientos relacionados con indiferencia, incomodidad, tranquilidad, felicidad por poder verificar el éxito por sí mismos y conmoción por el dolor ajeno. Es importante que los equipos quirúrgicos conozcan estos sentimientos $y$ reflexionen permanentemente, en el sentido de la calificación del cuidado preoperatorio. Cambios estructurales y de los procesos también pueden contribuir a una acogida más cuidadosa del paciente en el ambiente quirúrgico. 
Palabras Clave: Enfermería. Enfermería de Centro Quirúrgico. Sala de Recuperación. Cuidados preoperatorios.

\section{Introdução}

O período denominado perioperatório compreende um momento crítico para o indivíduo que será submetido a um procedimento cirúrgico, quando então estará exposto a riscos e complicações diversos, sendo necessária assistência especializada por parte das equipes médica e de enfermagem $^{(1-2)}$.

Um plano de cuidados fundamentado na comunicação, no treinamento e acompanhamento das equipes envolvidas permite intervenções adequadas no decorrer da experiência cirúrgica ${ }^{(2)}$. Os profissionais envolvidos devem trabalhar em harmonia utilizando de forma eficiente e eficaz seus conhecimentos, habilidades e competências com foco assistencial na qualidade, e livre de $\operatorname{danos}^{(3)}$.

O bem-estar do paciente cirúrgico é o objetivo principal no préoperatório, momento caracterizado por níveis variados de estresse e sentimentos que podem interferir em seu estado emocional ${ }^{(4)}$. Também fazem parte dessa etapa avaliações e checagens a partir das orientações da Organização Mundial da Saúde (OMS) para o que passou a ser denominado como cirurgia segura $^{(3)}$. A avaliação pré-operatória, que contempla várias dimensões além das protocolares e passa a se destacar ao procurar atender, além das necessidades fisiológicas, as psicológicas, espirituais e sociais ${ }^{(5)}$. O pós-operatório se destina a estabelecer um plano de recuperação que ressalte todas as preocupações inerentes a esse período ${ }^{(3)}$.

$\mathrm{Na}$ estrutura atualmente conhecida dos espaços cirúrgicos, na maior parte das instituições, encontra-se a sala de recuperação pós-anestésica (SRPA), quase sempre adjacente às salas de cirurgia, que teve seu início em 1801, na Inglaterra. Desde então, sua principal característica tem sido proporcionar um ambiente tranquilo, com equipamentos necessários para o suporte do paciente pós-operado, visando à recuperação desses indivíduos e a prevenção e o diagnóstico precoce de possíveis complicações relacionadas com os atos cirúrgico e anestésico ${ }^{(6)}$.

$$
\text { A resolução de } \mathrm{n}^{\circ} 50 \text { da }
$$

Diretoria Colegiada da Agência Nacional de Vigilância Sanitária, que dispõe sobre o Regulamento Técnico para planejamento, programação, elaboração e avaliação de projetos 
físicos de estabelecimentos assistenciais de saúde, preconiza a obrigatoriedade da existência, além da SRPA, de uma sala para recepção de pacientes no centro cirúrgico $^{(7)}$. Apesar disso, algumas instituições hospitalares também utilizam o espaço destinado ao período pós-operatório para acomodar indivíduos que se encontram na fase pré-operatória, o que pode aumentar o nível de ansiedade e estresse, como pode ser constatado em pesquisa que abordou a relação entre a orientação pré-operatória e a sensação de insegurança nos pacientes ${ }^{(8)}$.

Outro estudo sobre as percepções do cliente no pré-operatório em relação às condutas dos profissionais e assistência a eles prestada parte do pressuposto que anseios, medos e temores no préoperatório podem prejudicar a recuperação após o procedimento ${ }^{(9)}$. Urge, portanto, refletir sobre as maneiras de cuidar, as singularidades e subjetividade envolvidas que comprometam os profissionais de saúde com a humanização da assistência ${ }^{(10)}$.

$$
\text { Partindo-se do pressuposto }
$$
acima, os objetivos do presente estudo foram compreender a percepção do paciente que aguarda a sua cirurgia no mesmo espaço destinado aqueles já submetidos a procedimentos cirúrgicos e propor melhorias que visem à manutenção do bem-estar e a garantia da qualidade da assistência prestada no ambiente cirúrgico.

Torna-se relevante que profissionais envolvidos no cuidado perioperatório assumam o compromisso de qualificar a assistência em todos os momentos em que os indivíduos estão expostos em função de um ato cirúrgico, a partir do olhar desse indivíduo e não somente a partir do olhar de quem cuida. Enfermeiros lotados em centros cirúrgicos recebem e acolhem pacientes em estresse cirúrgico decorrente de experiências desconhecidas, e que necessitam de tranquilidade, conforto e orientações esclarecedoras. Vivenciar o préoperatório ao lado daqueles que foram operados e estão em observação na SRPA favorece sentimentos e angústias que precisam ser (re)conhecidos e refletidos para que sejam desencadeadas ações que qualifiquem o cuidado. Os resultados poderão contribuir igualmente para a formação de profissionais envolvidos com o cuidado perioperatório, bem como discutir, sob o ponto de vista gerencial, os procedimentos adotados, muitas vezes em função das condições estruturais, 
que possam melhor qualificar a assistência cirúrgica.

\section{Metodologia}

Trata-se de pesquisa exploratória com abordagem qualitativa realizada em um hospital universitário, filantrópico e sem fins lucrativos, na cidade de Belo Horizonte-MG. O hospital em questão tem contabilizado em torno de 70 mil consultas ambulatoriais e mais de seis mil internações anuais, nas mais diferentes áreas. No âmbito cirúrgico, cerca de 710 pacientes são submetidos a procedimentos mensalmente, com predominância de intervenções nas especialidades cirurgia geral e ortopédica. Desse total, cerca de 60 pacientes aguardam diária e sistematicamente pela cirurgia, no mesmo espaço físico que recebe os que já sofreram as intervenções.

Obteve-se o depoimento de 15 participantes que, entre abril e agosto de 2014, aguardaram pela cirurgia na SRPA. Foram incluídos os indivíduos com idade igual ou superior a 18 anos que aguardaram pelo procedimento cirúrgico na SRPA, juntamente com pacientes operados, e que concordaram em participar da pesquisa, por meio do termo de consentimento livre e esclarecido (TCLE) Foram excluídos aqueles que não possuíam capacidade física e/ou mental para responder às questões, e os que receberam alta hospitalar no mesmo dia, ou foram encaminhados diretamente para unidades de tratamento intensivo ao término da cirurgia, pois não estariam disponíveis, no dia seguinte, para a entrevista.

A coleta de dados ocorreu no dia imediatamente posterior à cirurgia, à beira do leito, gravada em mídia-player, após o consentimento que constava no TCLE.

$\mathrm{O}$ instrumento utilizado para a entrevista semiestruturada foi um roteiro contendo questões inerentes aos objetivos do estudo e outras perguntas complementares decorrentes da circunstância momentânea, permitindo que o depoente falasse abertamente sobre o que teria vivenciado durante o período pré-operatório, expondo sua opinião e sentimentos. $\mathrm{O}$ encerramento das entrevistas aconteceu ao ser detectada a saturação dos dados, quando o conteúdo expresso passou a ficar repetitivo e os objetivos haviam sido alcançados.

A análise dos depoimentos colhidos ocorreu após a sua transcrição, utilizando-se o referencial para análise do discurso de Bardin $^{(11)}$, no sentido de 
compreender a percepção dos indivíduos sobre a espera de sua cirurgia no mesmo espaço físico onde se encontravam pacientes já operados.

O rigor do estudo se deu por meio da obediência aos critérios de inclusão e exclusão; da realização das entrevistas sempre no mesmo momento pós-operatório; do seguimento do roteiro de questões previamente estabelecidas para o estudo e, finalmente, pelo fato da realização das entrevistas, transcrição e análise terem sido de exclusiva responsabilidade das pesquisadoras envolvidas. A investigação foi aprovada pelo Comitê de Ética em Pesquisa da Faculdade Ciências Médicas de Minas Gerais, Belo Horizonte-MG, sob o $\mathrm{n}^{\circ}$ $4.595 / 2014$.

\section{Resultados}

Participaram do estudo os 15 pacientes que atenderam aos critérios de inclusão e aguardaram pela cirurgia na SRPA junto com pacientes em pósoperatório imediato. Do total, 69,8\% $(n=37)$ eram do sexo masculino e $30,2 \%$ $(\mathrm{n}=16)$ do sexo feminino. Quanto à idade, o número de participantes foi ligeiramente maior nas faixas etárias de 41 a $60 \operatorname{anos}(35,8 \%, n=19)$ e de 21 a 40 $\operatorname{anos}(32,1 \%, \mathrm{n}=17)$.
Ao analisar as especialidades cirúrgicas a que foram submetidos, houve maior ocorrência de cirurgias ortopédicas $(43,4 \%, \mathrm{n}=23)$, seguidas pelas cirurgias gerais $(28,3 \%, \mathrm{n}=15)$. Os procedimentos cirúrgicos em ambas as especialidades foram classificados, em sua maioria, como de média complexidade.

Após a breve caracterização sociodemográfica e epidemiológica, realizou-se a análise dos depoimentos colhidos a partir de questões que abordavam os objetivos do presente estudo. Os depoentes foram identificados por meio da letra "P" (paciente), seguida de um número natural exclusivo para cada um deles, considerando a ordem cronológica das entrevistas realizadas.

A leitura cuidadosa dos aspectos abordados permitiu seu recorte de forma a agrupar aqueles semelhantes ou que, na sua essência, trouxessem o que de mais íntimo refletisse a experiência vivida. Cada pesquisador realizou o processo de leitura e recortes individualmente e, em seguida, reuniram-se para a análise conjunta. Tais recortes agrupados por semelhança no discurso puderam ser convertidos em cinco categorias que refletem sentimentos relacionados à indiferença, 
percepção de algum incômodo, experiências de tranquilidade, felicidade e comoção, os quais são apresentados e analisados a seguir.

\section{Sentimentos}

de

\section{normalidade/indiferença em relação à} estrutura

Alguns depoentes não manifestam descontentamento em relação à área física ou às suas condições:

"Não tive problemas. $O$ local não atrapalhou.”(P4) "Normal, o espaço... não muda muita coisa."(P3)

"Ambiente bem tranquilo, não teve nenhum transtorno aqui, não.”(P14)

Também afirmam que poderia não haver diferenças caso a espera ocorresse em outro local:

"Para mim foi tranquilo, não tem isso de falar que se eu estivesse em outro lugar seria melhor, não." (P15)

Outros indivíduos afirmaram, ainda, que esse seria o lugar ideal ou mais apropriado:

"Eu acho que é o lugar Ideal." (P8)

"Lá estava muito bom" (P1)

A atenção recebida no espaço conjunto também foi analisada como se estivesse de acordo com o que o Sistema Único de Saúde brasileiro pode oferecer:
"Achei normal, dentro do tratamento do SUS, eu achei normal." (P7)

Apesar da indiferença de alguns indivíduos em relação ao ambiente no momento pré-cirúrgico, na SRPA, outros se sentiram incomodados, o que está apresentado na categoria a seguir.

\section{Percepção de que algo causou incômodo}

Os depoentes demonstram certo desconforto em relação ao objeto de estudo: a espera conjunta. Tensão e medo aparecem nas expressões:

"Fiquei muito tensa. Mudei a posição que eu estava sentada, virei para o outro lado e fiquei de costas para o outro paciente. Eu estava de frente, fiquei de costas para ficar virada para a porta."(P14)

"Fiquei um pouco nervosa e... com frio na barriga.'(P5)

Também ficou evidente o constrangimento, a vergonha e o embaraço, em relação à exposição:

"Para mim foi péssimo, por causa da veste que a gente estava... a gente estava bem dizer nиa, tanto os homens como as mulheres. Nós ficamos com as costas de fora e misturados no pós (operatório), com gente para entrar, sacola na mão, porque eles não deixam os acompanhantes buscar aquela roupa que a 


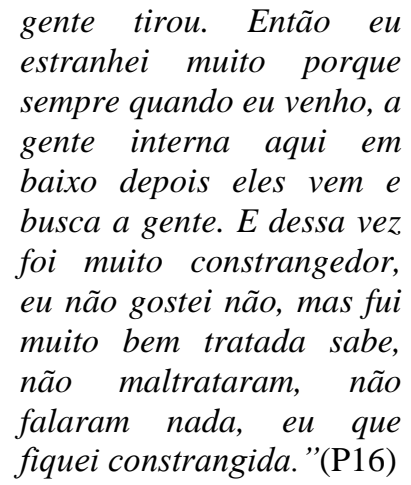

Chegam a sugerir outros ambientes com separação de pacientes:

"Tinha que ter um lugar para quem está esperando para fazer a cirurgia $e$ quem já fez a cirurgia."(P13)

"Seria bom outra sala, ali tem muita gente, todos saindo da operação, não é muito combinado."(P10)

A experiência de tranquilidade também foi percebida por eles. No entanto, esta categoria de depoimentos pode ter relação direta pelo fato das entrevistas se realizarem no dia seguinte, quando, supostamente, o momento crítico e as experiências de momento já se consolidavam.

\section{A tranquilidade como influência direta dos profissionais}

A segurança percebida nos profissionais foi considerada ferramenta importante que proporciona a sensação de tranquilidade e certeza do sucesso:

"Tranquilo. Vai muito do profissional que passa a tranquilidade ao paciente, até mesmo para a evolução no decorrer do procedimento."(P12).

"Foi muito bom que eu vi o atendimento de todos, eu mesmo tive que ser atendido lá porque eu passei mal, o atendimento foi rápido" (P9)

Estar em meio a outras pessoas que vivenciavam a mesma situação, além da tranquilidade gerada, emergiu em depoimentos que expressam felicidade, como pode ser constatado na próxima categoria.

\section{Felicidade por certificar-se do sucesso dos outros}

Os depoentes afirmaram um sentimento de felicidade em relação ao sucesso alheio, em contraponto ao medo citado:

$$
\begin{aligned}
& \text { "Aliviou pelo fato de já } \\
& \text { estar com medo, fiquei } \\
& \text { tranquilo quando a moça } \\
& \text { operada, conversou } \\
& \text { comigo."(P11) } \\
& \text { "É até interessante que a } \\
& \text { gente via que as pessoas já } \\
& \text { tinham operado, todo } \\
& \text { mundo estava bem ali na } \\
& \text { hora, foi um ânimo pra } \\
& \text { gente também" (P15) } \\
& \text { "Fique feliz quando vi que } \\
& \text { todo mundo fez e estava } \\
& \text { bem" (P6) }
\end{aligned}
$$

Enquanto esperam e observam os demais indivíduos já operados, surgem situações que emocionam, o que compõe o tema da próxima categoria.

\section{Sentimentos de comoção ao assistir a experiência do outro}

Alguns depoentes afirmaram ter ficado condoídos pela dor e sofrimento do próximo: 
"Fiquei com pena, porque chegou a menina, chorando."(P10)

"Tinha uma mulher que estava sentindo muita dor, estava chorando $e$

\section{Discussão} desesperada" (P2)

No período pré-operatório o paciente cirúrgico pode apresentar um alto nível de estresse que, por sua vez, pode torná-lo vulnerável e dependente. $\mathrm{O}$ ônus do estado emocional experimentado é indiscutível por ser um acontecimento crítico numa realidade desconhecida e assustadora ${ }^{(5)}$.

O estresse, muitas vezes, tem relação com a desinformação quanto aos procedimentos da cirurgia, da anestesia e sobre os cuidados a serem realizados, podendo estar relacionado com o grau de complexidade da cirurgia ${ }^{(4,8)}$. O medo também pode estar presente e, ter medo, é saber do que se tem medo ${ }^{(12)}$. Os fatores geradores de tais emoções são decorrentes do processo relacionado à doença que motiva a hospitalização, e à própria necessidade da cirurgia. Ambos geram afastamento familiar e das atividades do cotidiano ${ }^{(2)}$.

Por sua vez a indiferença expressa nos depoimentos aparece associada à expectativa em relação à prestação do serviço. Como se, previamente, o vivenciado significasse o que se esperava encontrar na $\operatorname{SRPA}^{(13)}$.
O fato de se afirmar que não se percebeu a influência da espera conjunta, pode representar uma tentativa de se acreditar nisso, para que realmente se concretize o que é desejado: o sucesso cirúrgico. O querer é querer algo já compreendido $^{(12)}$. Nesse sentido, afirmar que o local de espera cirúrgica não incomodou, também pode ter sido influenciado pelo resultado já supostamente positivo, considerando que as entrevistas aconteceram cerca de 24 horas após o momento vivido.

Ressalta-se que o bem-estar e a segurança do paciente no período que antecede o ato cirúrgico deve ser o foco dos profissionais ao se voltarem para as ações e cuidados específicos de cada cirurgia $^{(4,10)}$.

Para que isso aconteça toda a equipe deve estar atenta às questões mais íntimas dos pacientes cirúrgicos, as quais incluem exposição de partes do corpo, vestimentas reduzidas ou inadequadas, proximidade excessiva de outras pessoas estranhas e o cuidado com os objetos pessoais. O pudor do indivíduo é um cuidado básico para garantir o conforto social que deverá ser mantido em todas as situações, pois o paciente sempre se preocupa com a preservação da sua privacidade ${ }^{(14)}$. 
Estudo que caracterizou os cuidados de enfermagem realizados ao paciente cirúrgico no período préoperatório aponta o desafio da adequação de maior qualidade assistencial, levando-se em consideração suas reais necessidades, avaliadas individualmente, com a sua participação, e fundamentadas em bases teóricas ${ }^{(4,9)}$.

Torna-se evidente, pelos depoimentos, avaliar e considerar as expectativas do indivíduo em relação ao momento vivido, aos objetos pessoais e presença de familiares, assim como o número de pessoas durante o tempo de espera, os quais contribuem para a sensação de tranquilidade do paciente. Esse sentimento pôde ser observado pela calma, pelo menos aparente, dos indivíduos frente à situação, principalmente quando relacionada à certeza e segurança da assistência por bons profissionais, o que para eles é um fator que influencia os sentimentos vivenciados.

Em pesquisa que procurou analisar a cultura de segurança em centro cirúrgico de um hospital universitário do sul do Brasil, sob ponto de vista dos seus profissionais ${ }^{(1)}$, os resultados evidenciaram que os enfermeiros, em especial, tem papel importante na acolhida no ambiente cirúrgico dos indivíduos. Outro estudo afirma que o bem-estar do paciente deve constituir o principal objetivo dos profissionais, pois, no período préoperatório, é comum que desenvolvam sentimentos que afetam negativamente $o$ seu estado emocional ${ }^{(4)}$.

A presente investigação possibilitou perceber uma tendência, que independe do local da experiência, de acreditar no profissional assistente e esperar dele todas as informações que assegurem tranquilidade, bem-estar e redução da sensação de medo. Uma revisão da literatura sobre cuidados perioperatórios de enfermagem aponta que as orientações constituem elemento relevante que auxiliam paciente $\mathrm{e}$ família no enfrentamento do processo saúde-doença ${ }^{(2)}$.

O medo é certamente um dos sentimentos vivenciados em função do desconhecimento da situação futura a ser enfrentada. Pode causar insegurança e ansiedade $^{(15)}$. Aqueles que aguardam pelo procedimento cirúrgico tendem a sofrer transformações físicas, bem como psíquicas, como forma de reação imediata a pensamentos e/ou experiências cirúrgicas, o que pode acarretar sentimentos de fragilidade e incertezas. Em contrapartida, vivenciar o sucesso alheio frente ao ato cirurgico 
pode minimizar possíveis angústias e proporcionar o surgimento de emoções positivas como confiança e esperança, o que contribui para a sua melhora ${ }^{(16)}$. Percebeu-se que o sucesso cirúrgico dos companheiros de SRPA remete à sensação de alívio e esperança de que também a sua cirurgia também terá desfecho favorável.

Ao mesmo tempo que os indivíduos presenciam o sucesso cirúrgico alheio, assistem manifestações e emoções relacionadas aos aspectos biológicos como, por exemplo, a dor pós-operatória. Uma pesquisa ${ }^{(17)}$ demonstrou que, embora singular para quem a sente, a dor, por ser uma experiência humana e uma realidade coletiva, pode ser de certa forma, compartilhada. Frente à dor do outro, pode haver comoção com maior ou menor intensidade. Percebe-se comoção, e, talvez porissso apresentam sugestão de ambientes diferenciados, em função do que se viu e ouviu enquanto esperava.

A OMS, nas orientações destinadas aos procedimentos cirúrgicos seguros, enfatiza que independente da complexidade existem etapas e cuidados críticos, cada um deles com oportunidades para falhas que poderiam causar injúrias nos pacientes cirúrgicos $^{(3)}$. Torna-se relevante resgatar a base científica dos cuidados préoperatórios adequados a cada indivíduo, de acordo com suas necessidades ${ }^{(4)}$.

A percepção e experiência dos envolvidos tem sido cada vez mais utilizadas como indicadores na avaliação da qualidade da assistência prestada ${ }^{(13)}$. A análise decorrente de avaliações dos pacientes contribui para a reconstrução das práticas, melhorias institucionais e humanização do cuidado dispensado ${ }^{(9)}$.

Especificamente, na instituição onde se deu o presente estudo, não ocorrem visitas pré-operatórias do enfermeiro do centro cirúrgico para orientações sobre as condutas, espaços e procedimentos, e mesmo para o esclarecimento de dúvidas e anseios que amedrontam os pacientes.

Consideram-se pontos fortes da pesquisa o fato de levantar questões pertinentes ao cotidiano dos profissionais de saúde, especialmente aqueles envolvidos com o período perioperatório, tão aclamado nas publicações referentes à segurança do paciente. Além disso, os resultados podem embasar os processos de tomada de decisões que qualificam o cuidado cirúrgico.

Isso posto, avaliar práticas desenvolvidas são determinantes para 
que não se tornem banalizadas pela repetição e ritualização próprias do diaa-dia ${ }^{(8)}$, considerando que a percepção e experiência dos indivíduos tem sido cada vez mais utilizadas como indicadores na avaliação da qualidade da assistência $\operatorname{prestada}^{(13)}$. Autores reforçam que é preciso considerar as necessidades individuais para que $\mathrm{o}$ cuidar não se faça de forma mecânica e sem reflexão sobre o fazer ${ }^{(10)}$.

O estudo possui limitações por se tratar de abordagem qualitativa, no qual o número de participantes é reduzido, além de ter sido realizado em uma única instituição de saúde, o que pode não refletir outras realidades. Dessa forma, os resultados não tem a pretensão da generalização. A realização das entrevistas no dia seguinte à experiência vivida, em função da exiguidade de espaço na SRPA, quando os indivíduos já se encontravam na condição de pósoperados, pode ter modificado a real percepção do período pré-operatório na SRPA.

\section{Conclusão}

Os resultados apontaram a diversidade e a riqueza de sentimentos, percepções e experiências relacionados à espera da cirurgia no mesmo espaço físico destinado à recuperação pósoperatória. Os participantes demonstraram por meio dos depoimentos desde indiferença, sensação de estar incomodado com a experiência vivida, até tranquilidade pela certeza de uma boa assistência, a alegria por ter acontecido tudo como esperado, além dos sentimentos de comoção com a situação dos outros. A compreensão dessa percepção por parte dos profissionais de saúde deve ser considerada não somente em relação às questões legais, mas, principalmente para a qualificação e humanização do cuidado.

O local onde se deu o estudo possui espaço limitado que, no entanto, não impede adoção de medidas que modifiquem ou amenizem o convívio acima descrito. Considerando-se que parte dos objetivos do estudo envolvia propor melhorias locais poder-se-ia ressaltar que o estudo de novo lay out da área física disponível no ambiente cirúrgico poderia proporcionar a criação de um espaço exclusivo para o préoperatório imediato. Uma segunda possibilidade seria conduzir os pacientes para o Bloco Cirúrgico apenas no momento da cirurgia, diretamente do quarto para a sala cirúrgica, evitando-se assim, o encontro com pacientes 
operados e consequente espera conjunta na SRPA. E, por fim, na impossibilidade desse transporte para a sala de cirurgia no exato momento da cirurgia, a utilização de biombos fixos/móveis ou cortinas poderia minimizar $\mathrm{o}$ desconforto da espera simultânea e garantir maior privacidade e conforto para o indivíduo que aguarda sua cirurgia.

Como implicação prática ressalta-se a necessidade discussão periódica dos integrantes das equipes cirúrgicas que leve em consideração o olhar de quem é cuidado, a partir de levantamentos simples de avaliação dos indivíduos envolvidos.

Torna-se importante salientar que novos estudos dessa natureza são necessários e viriam a contribuir para a discussão das condições perioperatórias e das ações que envolvem um cuidado cirúrgico mais qualificado. É preciso refletir sobre as práticas desenvolvidas enquanto profissional de saúde. O uso de camisolas pouco privativas, o tempo de permanência em salas de préoperatório, a possibilidade de percepção de queixas de dor de outros pacientes, incluindo crianças, e as informações necessárias ao momento vivido poderiam se constituir em foco de tais pesquisas.

\section{Referências}

1. Da Corregio TC, Amante LN, Barbosa SFF. Avaliação da cultura de segurança do paciente em Centro Cirúrgico. Rev. SOBECC. 2014;19(2):67-73.

2. Guido LA, Goulart CT, Brum CN, Lemos AP, Umann J. Nursing perioperative care: an integrative review of literature. J. res.: fundam. care. online [Internet]. 2014 Out-Dez [citado 2015 Set 02];6(4),[S1]:1601-1609.

Disponível em: http://www.seer.unirio.br/index.php/cui dadofundamental/article/view/1554

3. Segundo desafio global para a segurança do paciente: cirurgias seguras salvam vidas (Manual de orientações para cirurgia segura). Ministério da Saúde; Agência Nacional de Vigilância Sanitária [Internet]. Brasília (Brasil). 2009. Disponível em http://bvsms.saude.gov.br/bvs/publicaco es/seguranca paciente cirurgia salva manual.pdf

4. Christóforo BEB, Carvalho DS. Cuidados de enfermagem realizados ao paciente cirúrgico no período préoperatório. Rev. esc. enferm. USP. 2009;43(1):14-22.

5. Santos MMB, Martins JCA, Oliveira LMN. A ansiedade, depressão e stresse no pre-operatorio do doente cirúrgico. Rev. Enf. Ref [Internet]. 2014 Dez [citado 2015 Set 02];IV(3):7-15. Disponível em: http://www.scielo.mec.pt/scielo.php?scr $\mathrm{ipt}=$ sci arttext \&pid=S087402832014000300002

6. Passos APP. O cuidado de enfermagem ao paciente cirúrgico frente ao ato anestésico. Persp. online: biol \& saúde [Internet]. 2012 Jul-Set [citado 2015 Mai 15];6(2):14-19. Disponível em: http://www.seer.perspectivasonline.com .br/index.php/biologicas_e saude/article /viewFile/202/119 
7. Regulamento Técnico para planejamento, programação, elaboração e avaliação de projetos físicos de estabelecimentos assistenciais de saúde [Internet]. Brasília (Brasil): ANVISA. 2002. Disponível em: http://portal.anvisa.gov.br/wps/content/ Anvisa+Portal/Anvisa/Inicio/Servicos+ de+Saude/Assunto+de+Interesse/Legisl acao/Projeto+fisico

8. Kruse MHL, Almeida MA, Keretzky KB, Rodrigues E, Silva FP, Schenini FS, et al. Orientação pré-operatória da enfermeira: lembranças de pacientes. Rev. Eletr. Enf [Internet]. 2009 Set [citado 2015 Jun 10];11(3):494-500. Disponível em: https://www.fen.ufg.br/fen_revista/v11/ n3/pdf/v11n3a05.pdf

9. Rocha DRR, Ivo OP. Assistência de enfermagem no pré-operatório e sua influência no pós-operatório. Rev. Enferm.Contemp. 2015;4(2):170-178.

10. Piexak DR, Ferreira CLL, Terra MG, Backes DS, Barlem JGT, Ilha S. Cuidado de enfermagem em unidade de internação cirúrgica: percepção dos pacientes. J. res.: fundam. care. online [Internet]. 2016 Jan-Mar [citado 2015 Set 02];8(1):3624-3632. Disponível em: http://www.seer.unirio.br/index.php/cui dadofundamental/article/view/3596/pdf 1765http://www.seer.unirio.br/index.p hp/cuidadofundamental/article/view/359 6/pdf 1766

11. Bardin L. Análise de Conteúdo. Lisboa: Edições 70; 2002.

\section{Participação dos autores}

Todos os autores participaram da elaboração do projeto, acompanharam a submissão / aprovação ao Comitê de Ética em Pesquisa, da organização e análise de dados e da revisão do manuscrito. Letícia Silva de Araújo, Poliana Rodrigues Vilas Boas, realizaram, além disso, coleta de dados juntos aos participantes do estudo.
12. Heidegger M. Ser e Tempo. Trad. de Márcia Sá Cavalcanti Schuback. Bragança Paulista: Universitária São Francisco; 2011. (Coleção Pensamento Humano).

13. Gouveia GC, Souza WV, Luna CF, Szwarcwald CL, Souza Junior PRB. Satisfação dos usuários com a assistência de saúde no estado de Pernambuco, Brasil. Cienc Saude Coletiva. 2011;16 (3):1849-1861.

14. Santos RM, Viana IRMN, Silva JR, Trezza MCSF. Leite JL. A enfermeira e a nudez do paciente. Rev. bras. enferm. 2010;63(6):877-886.

15. Silva JP, Garanhani ML. O significado do cuidado perioperatório para a criança cirúrgica. Rev. Eletr. Enf [Internet]. 2011 Abr-Jun [citado 2015 Mai 15];13(2):259-268. Disponível em: https://www.fen.ufg.br/fen revista/v13/ $\underline{\mathrm{n} 2 / \mathrm{v} 13 \mathrm{n} 2 \mathrm{a} 12 . \mathrm{htm}}$

16. Sampaio CEP, Ribeiro DA, Marta $C B$, Seabra Junior HC, Martins ERC, Francisco MTR. Fatores determinantes da ansiedade e mecanismos de coping em procedimentos cirúrgicos gerais. J. res: fundam. care.on line [Internet]. 2013 Out-Dez [citado 2015 Set 02];5(4):547-555. Disponível em: https://dialnet.unirioja.es/servlet/articulo ;jsessionid=94156184AB0A4F704F168 A2214B3F1A5.dialnet01?codigo $=4767$ $\underline{748}$

17. Sarti CA. A dor, o indivíduo e a cultura. Saúde e sociedade. 2001;10(1): 3-13

Recebido: 21.12.2016

Revisado: 09.04.2016

Aprovado: 02.05.2016 